\title{
ON THE ORDER OF GROUPS OF AUTOMORPHISMS*
}

BY

\section{GARRETT BIRKHOFF AND PHILIP HALL}

1. Introduction. Consider the following problem. Let $G$ be any group of finite order $g$, and let $A$ denote the group of the automorphisms of $G$. What can one infer about the order $a$ of $A$, simply from a knowledge of $g$ : in other words, to what extent is $a$ a numerical function of $g$ ?

The main known result relating to this problem is due to Frobenius. $\dagger$ It limits the orders of the individual elements of $A$ in terms of $g$, and hence tells which primes can be divisors of $a$.

The present paper is independent of the work of Frobenius, and presupposes only the theorems of Lagrange and Sylow. Its main result is the following

TheOREM 1. Let $G$ be any group of finite order $g$. Let $\theta(g)$ denote the order of the group of the automorphisms of the elementary Abelian group of order $g$, and let $r$ denote the number of distinct prime factors of $g$. Then the order a of the group $A$ of the automorphisms of $G$ is a divisor of $g^{r-1} \theta(g)$.

The function $\theta(g)$ is computed numerically from $g$ as follows. Write $g$ as the product $p_{1}^{n_{1}} p_{2}^{n_{2}} \cdots p_{r}^{n_{r}}$ of powers $p_{k}^{n_{k}}$ of distinct primes. Then

$$
\begin{aligned}
\theta\left(p_{k}^{n_{k}}\right) & =\left(p_{k}^{n_{k}}-1\right)\left(p_{k}^{n_{k}}-p\right) \cdots\left(p_{k}^{n_{k}}-p_{k}^{n_{k}-1}\right) \\
& =p_{k}^{n_{k}\left(n_{k}-1\right) / 2} \cdot\left(p_{k}-1\right)\left(p_{k}{ }^{2}-1\right) \cdots\left(p_{k}^{n_{k}}-1\right)
\end{aligned}
$$

and

$$
\theta(g)=\theta\left(p_{1}^{n_{1}}\right) \theta\left(p_{2}^{n_{2}}\right) \cdots \theta\left(p_{r}^{n_{r}}\right) .
$$

For example, $\theta(12)=\theta(3) \theta(4)=2 \cdot(3 \cdot 2)=12$.

One can strengthen Theorem 1 in special cases, by

THEOREM 2. If $G$ is solvable, then a is a divisor of $g \theta(g)$.

Theorem 3. If $G$ is "hypercentral," that is, the direct product of its Sylow subgroups, then a is a divisor of $\theta(g)$.

2. Preliminary lemmas. The following two statements are immediate corollaries of Lagrange's and Sylow's Theorems, respectively:

* Presented to the Society, December 26, 1933; received by the editors August 20, 1935.

$\dagger$ Über auflösbare Gruppen, II, Berliner Sitzungsberichte, 1895, p. 1030. Cf. Burnside's Theory of Groups, 1st edition, pp. 250-252. 
LEMma 1. Let $H$ be any group whose elements induce automorphisms homomorphically (i.e., many-one isomorphically) on a second group $G$. Then the index in $H$ of the subgroup "centralizing" $G$ (i.e., leaving every element of $G$ invariant) divides the order of the group of the automorphisms of $G$.

LEмма 2. Let $G$ be any group, and $r$ any positive integer. If the order of every prime-power subgroup of $G$ divides $r$, then the order of $G$ divides $r$.

As a further preliminary step, it is well to verify the somewhat less obvious

LeMma 3. Let $P$ be any group of prime-power order $p^{n}$, inducing substitutions homomorphically on $r=p^{\alpha} q$ letters $x_{1}, \cdots, x_{r}\left[p^{\alpha}\right.$ the highest power of $p$ dividing $r$ ]. Then there is a letter $x_{k}$ such that, if $S$ denotes the subgroup of substitutions of $P$ which omit $x_{k}$, the index of $S$ in $P$ divides $r$.

Let $S_{i}$ denote that subgroup of $P$ whose substitutions omit the letter $x_{i}$; by Lagrange's Theorem, the index of $S_{i}$ in $P$ is a power $p^{\beta(i)}$ of $p$. Hence the transitive system including $x_{i}$ contains exactly $p^{\beta(i)}$ letters. But the sum of the numbers of letters in the different transitive systems is not a multiple of $p^{\alpha+1}$; hence $\beta(i) \leqq \alpha$ for some $i=i_{0}$. Setting $S_{i}=S_{i_{0}}$, we have Lemma 3 .

Lemma $4 . \dagger$ Let $G$ be any group of prime-power order $p^{n}$. Then the order a of the group $A$ of the automorphisms of $G$ divides $\theta\left(p^{n}\right)=\left(p^{n}-1\right)\left(p^{n}-p\right) \cdots$ $\left(p^{n}-p^{n-1}\right)$.

By Lemma 2, it is sufficient to prove the result for every subgroup $Q$ of $A$ of prime-power order $q^{m}$. But given $Q$, one can define $Q_{1}>Q_{2}>Q_{3}>\cdots>Q_{r}=1$ and $S_{1}<S_{2}<S_{3}<\cdots<S_{r}=G$ recursively as follows:

(1) $Q_{1}$ is the group $Q$.

(2) Given $Q_{k}, S_{k}$ is the subgroup of the elements of $G$ "centralized" by $Q_{k}$ (i.e., invariant under every automorphism of $Q_{k}$ ).

(3) Given $Q_{k}$ and $S_{k}, Q_{k+1}$ is a proper subgroup of $Q_{k}$ whose index in $Q_{k}$ divides the number of elements in $G-S_{k}$.

The only questionable point in the existence of these subgroups concerns the possibility of (3); this is ensured by Lemma 3 .

Moreover multiplying together on one side the indices of the $Q_{k+1}$ in the $Q_{k}$, and on the other their multiples, the degrees of the $G-S_{k}$, one sees that $q^{m}$ divides the product of those factors $\left(p^{n}-p^{j}\right)$ corresponding to the orders

$\dagger$ A more delicate result implying this, but presupposing a study of the structure of groups of prime-power order, is given by $\mathrm{P}$. Hall in A contribution to the theory of groups of prime-power order, Proceedings of the London Mathematical Society, vol. 36 (1933), p. 37. 
of complexes $G-S_{k}$. Hence a fortiori $q^{m}$ divides $\theta\left(p^{n}\right)$, and the lemma is proved.

3. Proof of principal theorem. We are now in a position to prove Theorem 1.

Accordingly, let $G$ be any group of finite order $g$, let $g=p_{1}^{n_{1}} \cdots p_{r}^{n_{r}}$, let $\theta(g)$ denote the order of the group of the automorphisms of the elementary Abelian group of order $g$, and let $A$ (of order $a$ ) denote the group of the automorphisms of $G$.

By Sylow's Theorem, $G$ contains subgroups $S_{i}{ }^{i}$ of orders $p_{i}^{n_{i}}[i=1, \cdots, r$; $\left.j=1, \cdots, s_{i}\right]$. By Sylow's Theorem also, $\dagger s_{i}$ is the index in $G$ of the "normalizer" of any $S_{j}{ }^{i}$ (i.e., the set of elements $a \epsilon G$ such that $a S_{j}{ }^{i}=S_{j}{ }^{i} a$ ); hence, by Lagrange's Theorem and the fact that $S_{j}{ }^{i}$ is contained in its own normalizer, $s_{i}$ divides $g / p_{i}{ }^{n_{i}}$.

Again, the automorphisms of $G$ obviously permute the $S_{j}{ }^{i}$ of given order $p_{i}{ }^{n_{i}}$ homomorphically. Therefore, by iterated use of Lemma 3, any subgroup $Q$ of $A$ of prime-power order $q^{m}$ contains a subgroup $Q_{1}$ whose index in $Q$ divides the product $\prod_{i=1}^{r}\left(g / p_{i}^{n_{i}}\right)=g^{r-1}$, and which normalizes at least one $S_{j(i)}^{i}$ of each order $p_{i}{ }^{n_{i}}$. But by Lemma 1 and iterated use of Lemma $4, Q_{1}$ has a subgroup $Q^{*}$ whose index in $Q_{1}$ divides $\theta(g)$, and which "centralizes" $S_{j(1)}^{i}, \cdots, S_{j(r)}^{r}$ [i.e., leaves every element of these subgroups of $G$ invariant]. But the $S_{j(i)}^{i}$ generate $G$; hence $Q^{*}$ contains only the identity, and $q^{m}$ divides $g^{r-1} \theta(g)$.

Theorem 1 now follows from Lemma 2 and the fact that $Q$ was permitted to be an arbitrary group of prime-power order.

4. Special cases of solvable and hypercentral groups. The proofs of Theorems 2-3 are now immediate.

In fact, Theorem 3 is really a corollary of Lemma 4 . For the Sylow subgroups of a hypercentral group are characteristic. Denoting them by $S_{1}, \cdots, S_{r}$, one sees immediately that the group of the automorphisms of $G$ is the direct product of the groups of the automorphisms of the $S_{k}$, making the theorem obvious.

To prove Theorem 2, suppose that $G$ is solvable, and use the stronger known result, $\ddagger$ analogous to Sylow's Theorem, that $G$ contains subgroups of every index $p_{k}{ }^{{ }^{k}}$. Now in the proof of Theorem 1 presented in $\S 3$, if $q$ does not divide $g$, it is numerically evident that $q^{m}$ divides $\theta(g)$. Hence, by Lemma 2 , it is sufficient to show that if $q$ divides $g$, then $q^{m}$ divides $g \theta(g)$.

$\dagger$ More particularly, the part that states that the inner automorphisms of $G$ are transitive on the Sylow subgroups of any fixed order.

$\ddagger$ Cf. P. Hall, $A$ note on soluble groups, Journal of the London Mathematical Society, vol. 3 (1928), p. 99. 
But to say that $q$ divides $g$ is evidently to say that $q=p_{k}$ for suitable $k$; without loss of generality, we can assume $k=1$. In this case $Q$ normalizes some Sylow subgroup $S$ of $G$ of order $p_{1}^{{ }^{n_{1}}}$; this follows from Lemma 3 and the fact that the number of Sylow subgroups of order $p_{1}{ }^{{ }^{1}}$, being a divisor of $p_{2}{ }^{n_{2}} \cdots p_{r}{ }^{n_{r}}$, is not divisible by $q$. Moreover $Q$ has a subgroup $Q_{1}$ whose index in $Q$ divides $q^{n_{1}}$ [and hence $g$ ] which "normalizes" (i.e., leaves invariant) a subgroup $H$ of order $p_{2}^{n_{2}} \cdots p_{r}^{n_{r}}$ (and index $p_{1}^{n_{1}}$ ) in $G$; this follows from Lemma 3 and the fact that by Hall's Theorem cited above, the number of such subgroups $H$ is a divisor of $p_{1}^{n_{1}}$.

Finally, by Lemmas 1 and 4, the index in $Q_{1}$ of the subgroup $Q_{2}$ "centralizing" $S$ divides $\theta\left(q^{n_{1}}\right)$. And by induction on $g$, the index in $Q_{2}$ of the subgroup $Q^{*}$ "centralizing" $H$ divides $\left(p_{2}^{n_{2}} \cdots p_{r}^{n_{r}}\right) \cdot \theta\left(p_{2}{ }^{n_{2}} \cdots p_{r}^{n_{r}}\right)$, or, since it is by Lagrange's Theorem a power of $q=p_{1}$ and relatively prime to $p_{2}{ }^{n_{2}} \cdots p_{r}^{{ }_{r}}$, it divides $\theta\left(p_{2^{n_{2}}} \cdots p_{r^{n_{r}}}\right)$. But $S$ and $H$, if only by Lagrange's Theorem, generate $G$; hence $Q^{*}=1$. Combining, one sees that if $q$ divides $g$, then $q^{m}$ divides $g \theta\left(p_{1}^{n_{1}}\right) \theta\left(p_{2}^{n_{2}} \cdots p_{r}^{n_{r}}\right)$, that is, $g \theta(g)$. But this is just what we wished to prove.

5. Possible improvement of results. It is natural to ask what likelihood there is of improving the results expressed in Theorems 1-3.

It is well known that the least upper bound to the possible values of $a$ for fixed $g$ is at least $\theta(g)$; this is shown by the elementary Abelian group of order $g$. Consequently Theorem 3 is a best possible result. Moreover in general $\theta(g)$ is not a common multiple for the possible values of $a$, as is shown by the dihedral group of order six and many other groups of similar structure.

On the other hand, there is no known example of a group for which $a$ fails to divide $g \theta(g)$; this suggests the possibility of replacing $g^{r-1} \theta(g)$ in Theorem 1 by $g \theta(g)$, and omitting Theorem 2 altogether.

This leaves the determination of lower bounds and common divisors of $a$ in terms of $g$ unattempted. The cyclic groups of order $g$ should throw considerable light on this more trivial question.

Also, the case in which $G$ is simple would probably repay study.

HARVARD UNIVERSITY,

Cambridge, Mass.

King'S College,

Cambridge, England. 